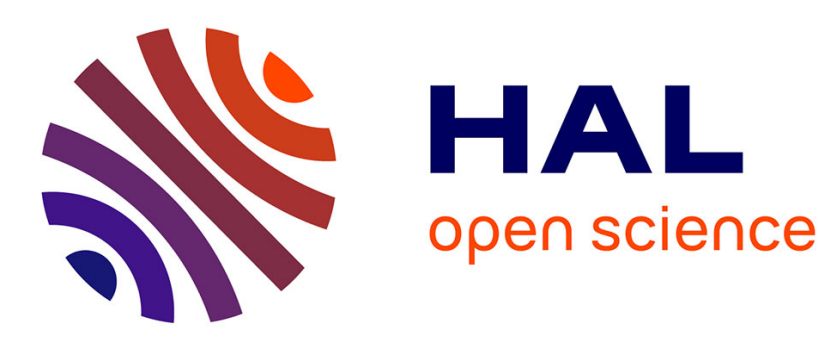

\title{
Robustness of Homogeneous Systems with Respect to Time-Varying Perturbations
}

Rios Hector, Denis Efimov, Wilfrid Perruquetti

\section{To cite this version:}

Rios Hector, Denis Efimov, Wilfrid Perruquetti. Robustness of Homogeneous Systems with Respect to Time-Varying Perturbations. [Research Report] Inria Lille; Non-A team. 2015. hal-01204800

\section{HAL Id: hal-01204800 \\ https://hal.inria.fr/hal-01204800}

Submitted on 24 Sep 2015

HAL is a multi-disciplinary open access archive for the deposit and dissemination of scientific research documents, whether they are published or not. The documents may come from teaching and research institutions in France or abroad, or from public or private research centers.
L'archive ouverte pluridisciplinaire HAL, est destinée au dépôt et à la diffusion de documents scientifiques de niveau recherche, publiés ou non, émanant des établissements d'enseignement et de recherche français ou étrangers, des laboratoires publics ou privés. 


\title{
Robustness of Homogeneous Systems with Respect to Time-Varying Perturbations
}

\author{
H. Ríos ${ }^{\dagger}$, D. Efimov ${ }^{\dagger *}$, A. Polyakov ${ }^{\dagger *}$ and W. Perruquetti ${ }^{\dagger}$
}

\begin{abstract}
The problem of stability robustness with respect to time-varying perturbations of a given frequency spectrum is studied applying homogeneity framework. The notion of finite-time stability over time intervals of finite length, i.e. short-finite-time stability, is introduced and used for that purpose. The results are applied to analyze the Super-Twisting algorithm behavior under some time-varying perturbations, and to demonstrate some robustness properties of the three-tank system. Some simulation examples illustrate these robustness properties.
\end{abstract}

\section{INTRODUCTION}

I $\mathrm{N}$ homogeneous time-invariant dynamical systems, the local and global behaviors are the same [1], and for stability/instability analysis, Lyapunov or Chetaev function of a homogeneous system can also be chosen homogeneous [2], [3], [4]. Such a strong symmetry has been found useful for stability analysis [5], [1], [6], [7], [3], approximation of system dynamics/solutions [8], [9], stabilization [10], [11], [12], [13], [14] and estimation [5], [9], [15]. Numerical analysis and design of homogeneous systems may be simpler since, for example, a Lyapunov function has to be constructed on a sphere only (on the whole state space it can extended using homogeneity).

In addition, the homogeneous systems have certain intrinsic robustness properties [16], [17], [18], [19]. For example, if a system is homogeneous (considering disturbance as an auxiliary variable) and asymptotically stable without disturbances, then it is robustly stable (input-to-state stable (ISS) or integral ISS (iISS) [19]). In some cases perturbations have a certain structure or features, robustness with respect to which it is necessary to establish. As an example the spectrum frequency of the perturbation can be considered, a dynamical system can be stable or have bounded solutions for some frequencies and be extremely sensitive to a particular frequency of exogenous disturbance (resonance phenomenon in dynamical systems).

In order to evaluate robustness with respect to frequencies the study of time-varying dynamical systems may be helpful. An extension of the homogeneity concept to time-varying systems has been given in [20], [21], where in the latter a reparametrization of time has also been required together with the state dilation. Recently the ideas of [20] have been extended in [22], where several stability results, uniformly in frequencies of the time-varying part, have been established. In this work this line of research is carried on, showing that if a homogeneous system is asymptotically stable for zero frequency (in the time-invariant case), then it inherits stable behavior for some sufficiently small frequencies (boundedness of the perturbation will not be asked).

Establishing stability properties, it is also important to quantify the rate of convergence in the system: exponential, asymptotic, finite-time or fixed-time [23], [24], [13], [25], [26], [27]. Frequently, the homogeneity theory is used to establish finite-time or fixed-time stability [10], [5], [28], [27]: for example, if a system is globally asymptotically stable and homogeneous of negative degree, then it is finite-time stable. In this work the problem of finite-time stability analysis will be also addressed for time-varying systems.

In some situations the stability may be analyzed on a limited time window, then the notion of short-time stability [29], [30], [31], [32] appears. This is the case studied in the present work, and the results are built on the intersection of finite-time and short-time stability.

The outline of this work is as follows. The preliminary definitions and the homogeneity framework are given in Section II. The robustness property for some class of homogeneous time-varying systems is presented in Section III. Application of the developed theory is considered in Section IV to analyze the Super-Twisting algorithm behavior under some time-varying perturbations, and to show some robustness properties of the three-tank system. Some concluding remarks are given in Section $\mathrm{V}$. The corresponding proofs for the main results are postponed to the Appendix.

${ }^{\dagger}$ Non-A team @ Inria, Parc Scientifique de la Haute Borne, 40 avenue Halley, 59650 Villeneuve d'Ascq, France and CRIStAL (UMRCNRS 9189), Ecole Centrale de Lille, BP 48, Cité Scientifique, 59651 Villeneuve-d'Ascq, France. Emails: hector.rios_barajas@inria.fr; denis.efimoveinria.fr; andrey.polyakov@inria.fr; wilfrid.perruquetti@ec-lille.fr

* Department of Control Systems and Informatics, Saint Petersburg State University of Information Technologies Mechanics and Optics (ITMO), Kronverkskiy av. 49, Saint Petersburg, 197101, Russia. 


\section{PRELIMINARIES}

Consider a time-varying differential equation [33]:

$$
\frac{d x(t)}{d t}=f(t, x(t)), t \geq t_{0}, t_{0} \in \mathbb{R}
$$

where $x(t) \in \mathbb{R}^{n}$ is the state vector; $f: \mathbb{R}^{n+1} \rightarrow \mathbb{R}^{n}$ is a continuous function with respect to $x$ and piecewise continuous with respect to $t, f(t, 0)=0$ for all $t \in \mathbb{R}$. It is assumed that solution of the system (1) for an initial state $x\left(t_{0}\right)=x_{0} \in \mathbb{R}^{n}$, where $t_{0} \in \mathbb{R}$ is the initial time, is denoted as $x\left(t, t_{0}, x_{0}\right)$ and it is defined on some finite time interval $\left[t_{0}, t_{0}+T\right)$ (the notation $x(t)$ is used to reference $x\left(t, t_{0}, x_{0}\right)$ if the origin of $x_{0}$ and $t_{0}$ is clear from the context).

Remark 1. Note that since it is not required that $f$ would be locally Lipschitz in $x$, then the system (1) may have non-unique solutions for some initial states. Following [26], assume that (1) has unique solutions for all initial states $x_{0} \in \mathbb{R}^{n} \backslash\{0\}$ except at the origin, and since $f(t, 0)=0$, for all $t \in \mathbb{R}$, in the origin always there is a solution $x\left(t, t_{0}, 0\right)=0$ for all $t \geq t_{0}$. In this case, by selecting only solution that stays at the origin, "weak" stability results can be presented.

Example 1. To illustrate the previous remark, consider a variant of (1)

$$
\dot{x}(t)=(|t|-1) x^{\frac{1}{3}}(t),
$$

with $x \in \mathbb{R}$, which for $x\left(t_{0}\right)=0$ has not a unique solution since a family of solutions could be given as follows

$$
x(t)= \begin{cases}0, & \forall \mathrm{t} \leq \mathrm{t}_{0}+\varepsilon, \\ {\left[\frac{t}{3}(|t|-2)-\frac{\left(t_{0}+\varepsilon\right)}{3}\left(\left|t_{0}+\varepsilon\right|-2\right)\right]^{\frac{3}{2}},} & \forall t>t_{0}+\varepsilon,\end{cases}
$$

for all $\left|t_{0}+\varepsilon\right| \geq 2$ and all $\varepsilon \in R_{+}$.

A continuous function $\sigma: \mathbb{R}_{+} \rightarrow \mathbb{R}_{+}$belongs to class $\mathcal{K}$ if it is strictly increasing and $\sigma(0)=0$; it belongs to class $\mathcal{K}_{\infty}$ if it is also unbounded. A continuous function $\beta: \mathbb{R}_{+} \times \mathbb{R}_{+} \rightarrow \mathbb{R}_{+}$belongs to class $\mathcal{K} \mathcal{L}$ if $\beta(\cdot, r) \in \mathcal{K}$ and $\beta(r, \cdot)$ is a strictly decreasing to zero for any fixed $r \in \mathbb{R}_{+}$. Denote a sequence of integers $1, \ldots, m$ as $\overline{1, m}$, and $|q|$ the Euclidean norm of a vector $q$.

\section{A. Stability definitions}

Let $\Omega, \Xi$ be open neighborhoods of the origin in $\mathbb{R}^{n}, 0 \in \Omega \subset \Xi$.

Definition 1. [33], [26] At the steady state $x=0$ the system (1) is said to be

a) Uniformly stable (US) if for any $\epsilon>0$ there is $\delta(\epsilon)>0$ such that for any $x_{0} \in \Omega$ with any $t_{0} \in \mathbb{R}$, if $\left|x_{0}\right| \leq \delta(\epsilon)$ then $\left|x\left(t, t_{0}, x_{0}\right)\right| \leq \epsilon$, for all $t \geq t_{0}$ and any $t_{0} \in \mathbb{R}$

b) Uniformly asymptotically stable (UAS) if it is US and for any $\kappa>0$ and $\epsilon>0$ there exists $T(\kappa, \epsilon) \geq 0$ such that for any $x_{0} \in \Omega$ with any $t_{0} \in \mathbb{R}$, if $\left|x_{0}\right| \leq \kappa$ then $\left|x\left(t, t_{0}, x_{0}\right)\right| \leq \epsilon$, for all $t \geq t_{0}+T(\kappa, \epsilon)$ and any $t_{0} \in \mathbb{R}$;

c) Uniformly finite-time stable (UFTS) if it is US and finite-time converging from $\Omega$, i.e. for any $x_{0} \in \Omega$ with any $t_{0} \in \mathbb{R}$ there exists $0 \leq T^{t_{0}, x_{0}}<+\infty$ such that $x\left(t, t_{0}, x_{0}\right)=0$ for all $t \geq T^{t_{0}, x_{0}}$. The function $T_{0}\left(t_{0}, x_{0}\right)=\inf \left\{T^{t_{0}, x_{0}} \geq 0\right.$ : $\left.x\left(t, t_{0}, x_{0}\right)=0 \forall t \geq T^{t_{0}, x_{0}}\right\}$ is called the settling time of the system (1).

If $\Omega=\mathbb{R}^{n}$, then the corresponding properties are called global uniform stability/asymptotic-stability/finite-time-stability of $x=0$.

Another version of uniform finite-time stability has also been proposed in [21].

In this work a special stability notion defined not for all $t_{0} \in \mathbb{R}$, as in Definition 1 , will be also of interest but for a compact interval of initial times $t_{0}$, and only on a fixed interval of time [29], [30], [31], [32]:

Definition 2. At the steady state $x=0$ the system (1) is said to be

a) Short-time stable (Short-TS) with respect to $\left(\Omega, \Xi, T^{0}, T_{f}\right)$ if for any $x_{0} \in \Omega,\left|x\left(t, t_{0}, x_{0}\right)\right| \in \Xi$ for all $t \in\left[t_{0}, T_{f}\right]$ for any $t_{0} \in\left[-T^{0}, T^{0}\right]$

b) Short-finite-time stable (Short-FTS) with respect to $\left(\Omega, \Xi, T^{0}, T_{f}\right)$ if it is Short-TS with respect to $\left(\Omega, \Xi, T^{0}, T_{f}\right)$ and finite-time converging from $\Omega$ with the convergence time $T^{t_{0}, x_{0}} \leq T_{f}$ for all $x_{0} \in \Omega$ and $t_{0} \in\left[-T^{0}, T^{0}\right]$;

c) Globally short-finite-time stable (GShort-FTS) if for any bounded set $\Omega \subset \mathbb{R}^{n}$ containing the origin there exist a bounded set $\Xi \subset \mathbb{R}^{n}, \Omega \subset \Xi$ and $T_{f}>0$ such that the system is Short-FTS with respect to $\left(\Omega, \Xi, T^{0}, T_{f}\right)$ for any $T^{0}$.

In [29], [30], [31], [32] the short-time stability is considered for a fixed initial time instant $t_{0}$ only.

Remark 2. Short-time stability [31] was originally called stability over a finite interval of time [29], [30], [32] which is related to the notion of practical stability [34], but the former notion is used here to avoid a confusion with finite-time stability from [23], [10], since both concepts of stability are used in the paper. 


\section{B. Homogeneity}

For any $r_{i}>0, i=\overline{1, n}$ and $\lambda>0$, define the dilation matrix $\Lambda_{r}(\lambda)=\operatorname{diag}\left\{\lambda^{r_{i}}\right\}_{i=1}^{n}$ and the vector of weights $r=$ $\left[r_{1}, \ldots, r_{n}\right]^{T}$.

For any $r_{i}>0, i=\overline{1, n}$ and $x \in \mathbb{R}^{n}$ the homogeneous norm can be defined as follows

$$
|x|_{r}=\left(\sum_{i=1}^{n}\left|x_{i}\right|^{\frac{\rho}{r_{i}}}\right)^{\frac{1}{\rho}}, \rho=\prod_{i=1}^{n} r_{i} .
$$

For all $x \in \mathbb{R}^{n}$, its Euclidean norm $|x|$ is related with the homogeneous one:

$$
\underline{\sigma}_{r}\left(|x|_{r}\right) \leq|x| \leq \bar{\sigma}_{r}\left(|x|_{r}\right),
$$

for some $\underline{\sigma}_{r}, \bar{\sigma}_{r} \in \mathcal{K}_{\infty}$. In the following, due to this "equivalence", stability analysis with respect to the norm $|x|$ will be substituted with analysis for the norm $|x|_{r}$. The homogeneous norm has an important property that is $\left|\Lambda_{r}(\lambda) x\right|_{r}=\lambda|x|_{r}$ for all $x \in \mathbb{R}^{n}$. Define $\mathbb{S}_{r}=\left\{x \in \mathbb{R}^{n}:|x|_{r}=1\right\}$.

Definition 3. [2] The function $g: \mathbb{R}^{n} \rightarrow \mathbb{R}$ is called $r$-homogeneous $\left(r_{i}>0, i=\overline{1, n}\right)$, if for any $x \in \mathbb{R}^{n}$ the relation

$$
g\left(\Lambda_{r}(\lambda) x\right)=\lambda^{\nu} g(x),
$$

holds for some $\nu \in \mathbb{R}$ and all $\lambda>0$.

The function $f: \mathbb{R}^{n} \rightarrow \mathbb{R}^{n}$ is called $r$-homogeneous $\left(r_{i}>0, i=\overline{1, n}\right)$, if for any $x \in \mathbb{R}^{n}$ the relation

$$
f\left(\Lambda_{r}(\lambda) x\right)=\lambda^{\nu} \Lambda_{r}(\lambda) f(x),
$$

holds for some $\nu \geq-\min _{1 \leq i \leq n} r_{i}$ and all $\lambda>0$. In both cases, the constant $\nu$ is called the degree of homogeneity.

A dynamical system

$$
\dot{x}(t)=f(x(t)), t \geq 0,
$$

is called $r$-homogeneous of degree $\nu$ if this property is satisfied for the vector function $f$ in the sense of Definition 3. An advantage of homogeneous systems described by nonlinear ordinary differential equations is that any of its solution can be obtained from another solution under the state dilation and a suitable time rescaling:

Proposition 1. [1] Let $x: \mathbb{R}_{+} \rightarrow \mathbb{R}^{n}$ be a solution of the r-homogeneous system (3) with the degree $\nu$ for an initial state $x_{0} \in \mathbb{R}^{n}$. For any $\lambda>0$ define $y(t)=\Lambda_{r}(\lambda) x\left(\lambda^{\nu} t\right)$ for all $t \geq 0$, then $y(t)$ is also a solution of (3) with the initial state $y_{0}=\Lambda_{r}(\lambda) x_{0}$.

Homogeneous systems possess certain robustness with respect to external disturbances. For example, consider the system

$$
\dot{x}(t)=F(x(t), d(t)), t \geq 0,
$$

where $x(t) \in \mathbb{R}^{n}$ is the state, $d(t) \in \mathbb{R}^{m}$ is external disturbance, $d: \mathbb{R} \rightarrow \mathbb{R}^{m}$ is measurable and essentially bounded function of time, $F: \mathbb{R}^{n+m} \rightarrow \mathbb{R}^{n}$ is locally Lipschitz continuous.

Theorem 1. [19] Let $F\left(\Lambda_{r}(\lambda) x, \Lambda_{\tilde{r}}(\lambda) d\right)=\lambda^{\nu} \Lambda_{r}(\lambda) F(x, d)$ for all $x \in \mathbb{R}^{n}, d \in \mathbb{R}^{m}$ and all $\lambda>0$ with the weights $r=\left[r_{1}, \ldots, r_{n}\right]>0, \tilde{r}=\left[\tilde{r}_{1}, \ldots, \tilde{r}_{m}\right] \geq 0$ with a degree $\nu \geq-\min _{1 \leq i \leq n} r_{i}$. Assume that the system (4) is globally asymptotically stable for $d=0$, then the system (4) is

- ISS if $\tilde{r}_{\min }>0$, where $\tilde{r}_{\min }=\min _{1 \leq j \leq m} \tilde{r}_{j}$;

- iISS if $\tilde{r}_{\min }=0$ and $\nu \leq 0$.

\section{Homogeneity for time-varying systems}

The weighted homogeneity property, introduced for time-invariant systems in Definition 3, is understood for the time-varying systems (1) in the following sense.

Definition 4. [20] The function $g: \mathbb{R}^{n+1} \rightarrow \mathbb{R}$ is called $r$-homogeneous $\left(r_{i}>0, i=\overline{1, n}\right)$, if for any $x \in \mathbb{R}^{n}$ and $t \in \mathbb{R}$ the relation

$$
g\left(t, \Lambda_{r}(\lambda) x\right)=\lambda^{\nu} g(t, x)
$$

holds for some $\nu \in \mathbb{R}$ and all $\lambda>0$.

The function $f: \mathbb{R}^{n+1} \rightarrow \mathbb{R}^{n}$ is called $r$-homogeneous $\left(r_{i}>0, i=\overline{1, n}\right)$, if for any $x \in \mathbb{R}^{n}$ and $t \in \mathbb{R}$ the relation

$$
f\left(t, \Lambda_{r}(\lambda) x\right)=\lambda^{\nu} \Lambda_{r}(\lambda) f(t, x),
$$

holds for some $\nu \geq-\min _{1 \leq i \leq n} r_{i}$ and all $\lambda>0$. 
Thus, in the time-varying case (1) the homogeneity can be verified interpreting $t$ as a constant parameter. Consider also the following modification of the system (1):

$$
\frac{d x(t)}{d t}=f(\omega t, x(t)), t \geq t_{0}, t_{0} \in \mathbb{R},
$$

for some $\omega>0$. The parameter $\omega$ represents the frequency of time-varying part of the system. For an initial state $x_{0} \in \mathbb{R}^{n}$ denote the corresponding solution of (5) as $x_{\omega}\left(t, t_{0}, x_{0}\right)$, thus $x\left(t, t_{0}, x_{0}\right)=x_{1}\left(t, t_{0}, x_{0}\right)$. In this case the following extension of Proposition 1 is provided.

Proposition 2. [22], [20] Let $x\left(t, t_{0}, x_{0}\right)$ be a solution of the r-homogeneous system (1) with the degree $\nu$ for an initial state $x_{0} \in \mathbb{R}^{n}$. For any $\lambda>0$ the system (5) with $\omega=\lambda^{\nu}$ has a solution $y\left(t, t_{0}, y_{0}\right)=\Lambda_{r}(\lambda) x\left(\lambda^{\nu} t, \lambda^{\nu} t_{0}, x_{0}\right)$ for all $t \geq t_{0}$ with the initial state $y_{0}=\Lambda_{r}(\lambda) x_{0}$.

It is a well known fact that for linear time-varying systems (i.e. homogeneous systems of degree $\nu=0$ ) that its stability for some $\omega$ does not imply stability for all $\omega \in(0,+\infty)$. Surprisingly, for nonlinear homogeneous time-varying systems with degree $\nu \neq 0$ this is not the case:

Lemma 1. [22], [20] Let the system (1) be r-homogeneous with degree $\nu \neq 0$ and GUAS, i.e. there is $\beta \in \mathcal{K} \mathcal{L}$ such that

$$
\left|x\left(t, t_{0}, x_{0}\right)\right|_{r} \leq \beta\left(\left|x_{0}\right|_{r}, t-t_{0}\right), \quad \forall t \geq t_{0},
$$

for any $x_{0} \in \mathbb{R}^{n}$ and any $t_{0} \in \mathbb{R}$. Then, (5) is GUAS for any $\omega>0$ and

$$
\left|x_{\omega}\left(t, t_{0}, x_{0}\right)\right|_{r} \leq \beta_{\omega}\left(\left|x_{0}\right|_{r}, t-t_{0}\right), \quad \forall t \geq t_{0},
$$

for any $x_{0} \in \mathbb{R}^{n}$ and any $t_{0} \in \mathbb{R}$, where $\beta_{\omega}(s, t)=\omega^{1 / \nu} \beta\left(\omega^{-1 / \nu} s, \omega t\right)$.

Thus, it is shown in Lemma 1 that the rate of convergence will be scaled by $\omega$, then the time of transients in these systems is predefined by the time-varying part, which is not the case for the degree $\nu=0$, where the rate of convergence cannot be modified by $\omega$. Several useful consequences of Proposition 2 and Lemma 1 can be formulated.

Corollary 1. [22] Let the system (1) be r-homogeneous with degree $\nu=0$ and UAS into the set $\Omega=B_{\rho}=\left\{x \in \mathbb{R}^{n}:|x|_{r} \leq \rho\right\}$ for some $0<\rho<+\infty$, then (1) is GUAS.

Corollary 2. [22] Let the system (5) be r-homogeneous with degree $\nu \neq 0$ and UAS into the set $\Omega=B_{\rho}=\left\{x \in \mathbb{R}^{n}:|x|_{r} \leq \rho\right\}$ for a fixed $0<\rho<+\infty$ for any $\omega>0$, then (5) is GUAS for any $\omega>0$.

Corollary 3. [22] Let the system (5) be r-homogeneous with degree $\nu>0$ and UAS into the set $B_{\rho}$ for some $0<\rho<+\infty$ for any $0 \leq \omega \leq \omega_{0}$ with $0<\omega_{0}<+\infty$, then (5) is GUAS for any $\omega>0$.

Corollary 4. [22] Let the system (5) be r-homogeneous with degree $\nu<0$ and UAS with respect to the set $B_{\rho}$ for some $0<\rho<+\infty$ for any $0 \leq \omega \leq \omega_{0}$ with $0<\omega_{0}<+\infty^{1}$, then (5) is GUAS for any $\omega>0$.

\section{Main Result: Short-Finite-Time Stability}

In this section the problem of stability robustness is considered with respect to time-varying part. Assuming that for $\omega=0$ the system (5) is stable and homogeneous, it will be shown that in this case a certain stability will be preserved for a frequency spectrum sufficiently close to zero. All proofs are given in the Appendix. The following continuity restriction is imposed on $f$ in (5).

Assumption 1. For (5), there exists a function $\sigma \in \mathcal{K}_{\infty}$ such that

$$
\sup _{\xi \in \mathbb{S}_{r}}|f(\tau, \xi)-f(0, \xi)| \leq \sigma(|\tau|), \quad \forall \tau \in \mathbb{R} .
$$

In the following, two cases will be considered depending on the sign of homogeneity degree.

Lemma 2. Let the system (5) be r-homogeneous with degree $\nu>0$, asymptotically stable for $\omega=0$, and Assumption 1 be satisfied. Then for any $\rho>0$ and $T^{0}>0$ there is $\omega_{0}>0$ such that the set $B_{\rho}$ is reached by any solution of (5) with $\omega \in\left[-\omega_{0}, \omega_{0}\right]$ for all initial states $x_{0} \notin B_{\rho}$ at initial times $t_{0} \in\left[-T^{0}, T^{0}\right]$.

Note that the result of Lemma 2 does not claim that the set $B_{\rho}$ is stable or invariant since, in general, a trajectory can next leave $B_{\rho}$ and return to infinity. Such a behavior is possible since the term proportional to $\omega t$ may be asymptotically unbounded in the lemma conditions. This result can be interpreted as a short-finite-time stability of $B_{\rho}$.

\footnotetext{
${ }^{1}$ In this case for each $0 \leq \omega \leq \omega_{0}$, any $\varepsilon>0$ and $\kappa \geq 0$, there is $0 \leq T_{\kappa, \varepsilon}^{\omega}<+\infty$, such that $\left|x_{\omega}\left(t, t_{0}, x_{0}\right)\right|_{r} \leq \rho+\varepsilon$, for all $t \geq t_{0}+T_{\kappa, \varepsilon}^{\omega}$, for any $x_{0} \in B_{\kappa}$, and $\left|x\left(t, t_{0}, x_{0}\right)\right|_{r} \leq \bar{\sigma}_{\omega}\left(\left|x_{0}\right|_{r}\right)$, for all $t \geq t_{0}$, for some function $\sigma_{\omega} \in \mathcal{K}$, for all $t_{0} \in \mathbb{R}$.
} 
Lemma 3. Let the system (5) be $r$-homogeneous with degree $\nu<0$, asymptotically stable for $\omega=0$, and Assumption 1 be satisfied. Then for any $\rho>0$ and $T^{0}>0$ there are $\omega_{0}>0, \vartheta \geq 1$ and $T_{f}>T^{0}$ such that (5) with $\omega \in\left[-\omega_{0}, \omega_{0}\right]$ is Short-FTS at the origin with respect to $\left(B_{\rho}, B_{\vartheta}, T^{0}, T_{f}\right)$.

For Lemma 2, since $f(t, 0)=0$ for any $t \in \mathbb{R}$, there is a solution which stays at the origin for all $t \geq T_{f}$, then a kind of standard finite-time stability notion can be recovered in "weak" sense, for a part of solutions.

Remark 3. If it is assumed that the system (5) is periodical in time, i.e. $f(t, x)=f(t+\mathcal{T}, x)$ for all $x \in \mathbb{R}^{n}$, all $t \in \mathbb{R}$ and some $\mathcal{T}>0$, then initial times can be selected always in the interval $t_{0} \in[-0.5 \mathcal{T}, 0.5 \mathcal{T}]$. Therefore, Lemma 2 implies that for any $\rho>0$ there is $\omega_{0}>0$ such that the set $B_{\rho}$ is uniformly reached by any solution of (5) with $\omega \in\left[-\omega_{0}, \omega_{0}\right]$ for initial states $x_{0} \notin B_{\rho}$, while Lemma 3 ensures Short-FTS of (5) with $\omega \in\left[-\omega_{0}, \omega_{0}\right]$ uniformly in $T^{0}$.

The result of Lemma 3, under additional mild conditions on the system robustness and boundedness of the time-varying perturbation, can be extended to global short-finite-time stability. For this reason the following class of functions is introduced for $\varrho \in \mathcal{K}$ and $\delta>0$ :

$$
\begin{aligned}
\mathcal{L}_{\varrho, \delta}^{m}= & \left\{d: \mathbb{R} \rightarrow \mathbb{R}^{m}:|d(s)| \leq \varrho(s) \forall s \geq 0 ;\right. \\
& \exists \tau>0: d(s)=0, \forall|s| \geq \tau ; \\
& \left.\max \left\{|d|_{1},|d|_{\infty}\right\} \leq \delta\right\},
\end{aligned}
$$

where

$$
|d|_{1}=\int_{-\infty}^{+\infty}|d(t)| d t,|d|_{\infty}=\sup _{t \in \mathbb{R}}|d(t)| .
$$

Theorem 2. Let conditions of Theorem 1 be satisfied for (4) with $\nu<0$ and $\tilde{r}=[0, \ldots, 0]$. Then there exist $\varrho \in \mathcal{K}$ and $\delta>0$ such that (4) is GShort-FTS provided that $d \in \mathcal{L}_{\varrho, \delta}^{m}$, and $d(t)=d(t+\mathcal{T})$ for all $t \in \mathbb{R}$, with $d(t) \neq 0$ and some $\mathcal{T}>0$.

This theorem illustrates, in particular, the features of resonance behavior in nonlinear systems: it is dependent on the amplitude and frequency of the excitation, since selection of the gain function $\varrho$ is related with the frequency restriction. For linear systems, i.e. with $\nu=0$, there is dependence on frequency only.

Theorem 2 provides also an extension of Theorem 4.1 in [26]:

Corollary 5. Let the system in (5) possess a Lyapunov function $V: \mathbb{R} \times \Omega \rightarrow \mathbb{R}_{+}$, where $0 \in \Omega \subset \mathbb{R}^{n}$ is an open neighborhood of the origin, such that for all $x \in \Omega$ and $t \in \mathbb{R}$

$$
\begin{gathered}
\alpha_{1}(|x|) \leq V(t, x) \leq \alpha_{2}(|x|), \quad \alpha_{1}, \alpha_{2} \in \mathcal{K}_{\infty} ; \\
\dot{V}(t, x) \leq-\alpha V^{\eta}+k(\omega t) V^{\eta}, \quad \alpha>0, \eta \in(0,1),
\end{gathered}
$$

for a continuous $k: \mathbb{R} \rightarrow \mathbb{R}, k(0)=0$. Then for any $T^{0}>0$ there exist $\omega_{0}>0$ such that for $|\omega| \leq \omega_{0}$ the system (5) is Short-FTS with respect to $\left(\Omega, \Xi, T^{0}, T_{f}\right)$ for some $\Omega \subset \Xi \subset \mathbb{R}^{n}$ and $T_{f} \geq T^{0}$.

If $\Omega=\mathbb{R}^{n}$ and $k(t)$ is periodic, then there exist $\omega_{0}>0$ and $\delta>0$ such that for $k \in \mathcal{L}_{\varrho, \delta}^{1}, \varrho(s)=\sup _{|t| \leq s} k\left(\omega_{0} t\right)$ the system (5) is GShort-FTS.

Note that contrarily to [26] there is no requirement on the sign of the function $k$ in Corollary 5.

Example 2. Consider a system

$$
\dot{x}(t)=-\sqrt{|x(t)|} \operatorname{sign}(x(t))+\delta \sin (\omega t) \sqrt{|x(t)|},
$$

that is an example of the system considered in Corollary 5 with $\eta=0.5$ and $\nu=-0.5$. Simulating this system on the time interval $[0,100]$ (by iISS property this system has bounded solutions for any $\delta$ and $\omega$ on this interval) for different values of $(\omega, \delta)$ and calculating the map

$$
a(\omega, \delta)=\log \left[\max _{50 \leq t \leq 100}|x(t)|\right],
$$

the results given in Fig. 1 are obtained. As it can be seen from this figure, the system is converging to the origin for a small amplitude $\delta$ and for a small values of the frequency $\omega$, as it has been proven in Theorem 2 and Corollary 5. The "resonance" happens for particular combinations of $\delta$ and $\omega$. 


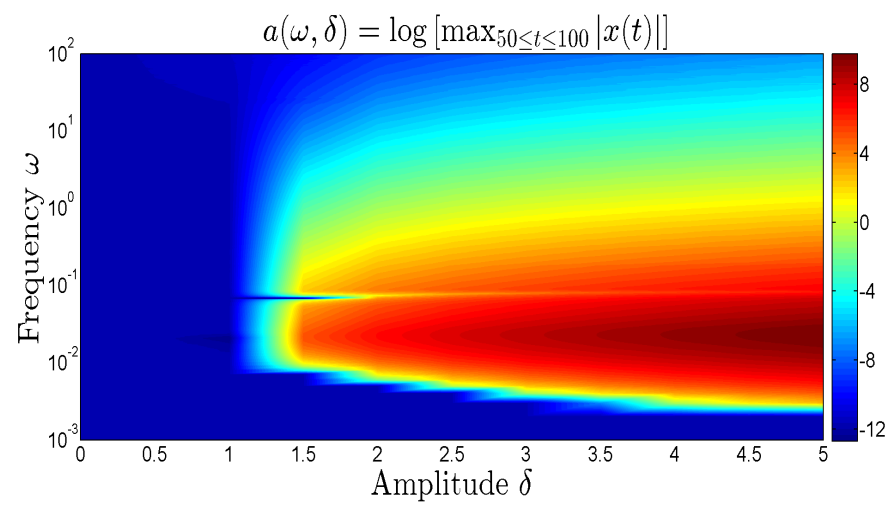

Figure 1. Illustration for Corollary 5 applied to Example 2.

Remark 4. Note that the results given in this section can be extended for dynamical systems (5) with discontinuous right-hand side through the ISS/iISS results given in Theorem 1 [19], and the homogeneity notion for differential inclusions [35].

\section{ApPliCATiON}

\section{A. Super-Twisting Algorithm}

Let us introduce the following time-varying system

$$
\dot{x}(t)=K(\omega t) u(t)+\phi(\omega t, \delta),
$$

where $x \in \mathbb{R}$ is the state, $u \in \mathbb{R}$ is the control input, $\phi: \mathbb{R} \times \mathbb{R} \rightarrow \mathbb{R}$ represents some bounded disturbances, i.e. $|\phi(\omega t, \delta)| \leq \delta$, for all $\omega, \delta \in \mathbb{R}$, frequency and amplitude, respectively; and all $t \in \mathbb{R}_{+}$; and $K: \mathbb{R} \rightarrow \mathbb{R}$ is an unknown time-varying function such that $K(0)>0$. It is worth mentioning that function $K$ can change its sign and therefore the sign of $u$.

Consider the Super-Twisting algorithm (STA) [36]

$$
\begin{aligned}
u(t) & =-k_{1}\lceil x(t)\rfloor^{\frac{1}{2}}+u_{1}(t), \\
\dot{u}_{1}(t) & =-k_{2}\lceil x(t)\rfloor^{0},
\end{aligned}
$$

where $\lceil\cdot\rfloor^{\gamma} \doteq|\cdot|^{\gamma} \operatorname{sign}(\cdot)$, and $k_{1}, k_{2}>0$ are positive constants. Then, let us consider the closed-loop system, i.e.

$$
\begin{aligned}
\dot{x}(t) & =K(\omega t)\left(-k_{1}\lceil x(t)\rfloor^{\frac{1}{2}}+u_{1}(t)\right)+\phi(\omega t, \delta), \\
\dot{u}_{1}(t) & =-k_{2}\lceil x(t)\rfloor^{0} .
\end{aligned}
$$

Define the variable $z(t)=K(\omega t) u_{1}(t)+\phi(\omega t, \delta)$. Hence, the closed-loop system may be rewritten as follows

$$
\begin{aligned}
& \dot{x}(t)=-K(\omega t) k_{1}\lceil x(t)\rfloor^{\frac{1}{2}}+z(t), \\
& \dot{z}(t)=-K(\omega t) k_{2}\lceil x(t)\rfloor^{0}+d(t),
\end{aligned}
$$

where $d(t)=\dot{K}(\omega t) u_{1}(t)+\dot{\phi}(\omega t, \delta)$. Therefore, let us assume that $K$ and $\phi$ are such that $d(0)=0$. Note that the previous closed-loop system has the form (5). Let us apply the results given by Lemma 3 in order to prove that the closed-loop system (6)-(7) is Short-FTS.

System (6)-(7) is $r$-homogeneous with degree $\nu=-0.5$ for $r=\left[r_{1}, r_{2}\right]=[1,0.5]$. Fig. 2 depicts system trajectories for different values of $\omega$ with fixed values of $\delta, k_{1}$ and $k_{2}$, which depict some fractality properties.

For the case $\omega=0$, system (6)-(7) becomes the classic STA, i.e.

$$
\begin{aligned}
& \dot{x}(t)=-\alpha k_{1}\lceil x(t)\rfloor^{\frac{1}{2}}+z(t), \\
& \dot{z}(t)=-\alpha k_{2}\lceil x(t)\rfloor^{0}
\end{aligned}
$$

where $\alpha=K(0)>0$. It has been proved in [36] (recently in [37]) that STA possesses convergence in finite-time. Since $K$ and $\phi$ are locally Lipschitz, and $d(0)=0$, Assumption 1 holds. Thus, all the conditions for Lemma 3 are satisfied and then system (6)-(7) is Short-FTS with respect to $\left(B_{\rho}, B_{\vartheta \rho}, T^{0}, T_{f}\right)$ for any $\rho>0, \vartheta \geq 1$ and $T_{f}>T^{0}>0$.

Let us consider that $K(\omega t)=1.5 \sin (\omega t)+1.1, \phi(\omega t, \delta)=\delta(\sin (2 \omega t)+\cos (\omega t))$, and thus $d(t)=1.5 \omega \cos (\omega t) u_{1}(t)+$ $\delta(2 \omega \cos (2 \omega t)-\omega \sin (\omega t))$, with $u_{1}(0)$ such that $d(0)=0$, and that the value of the STA gains are fixed, i.e. $k_{1}=20$ and $k_{2}=100$. Simulating the closed-loop system on the time interval $[0,100]$ for different values of $(\omega, \delta)$ and calculating $a(\omega, \delta)$ (previously defined in Example 2), the results given in Fig. 3 are obtained. 

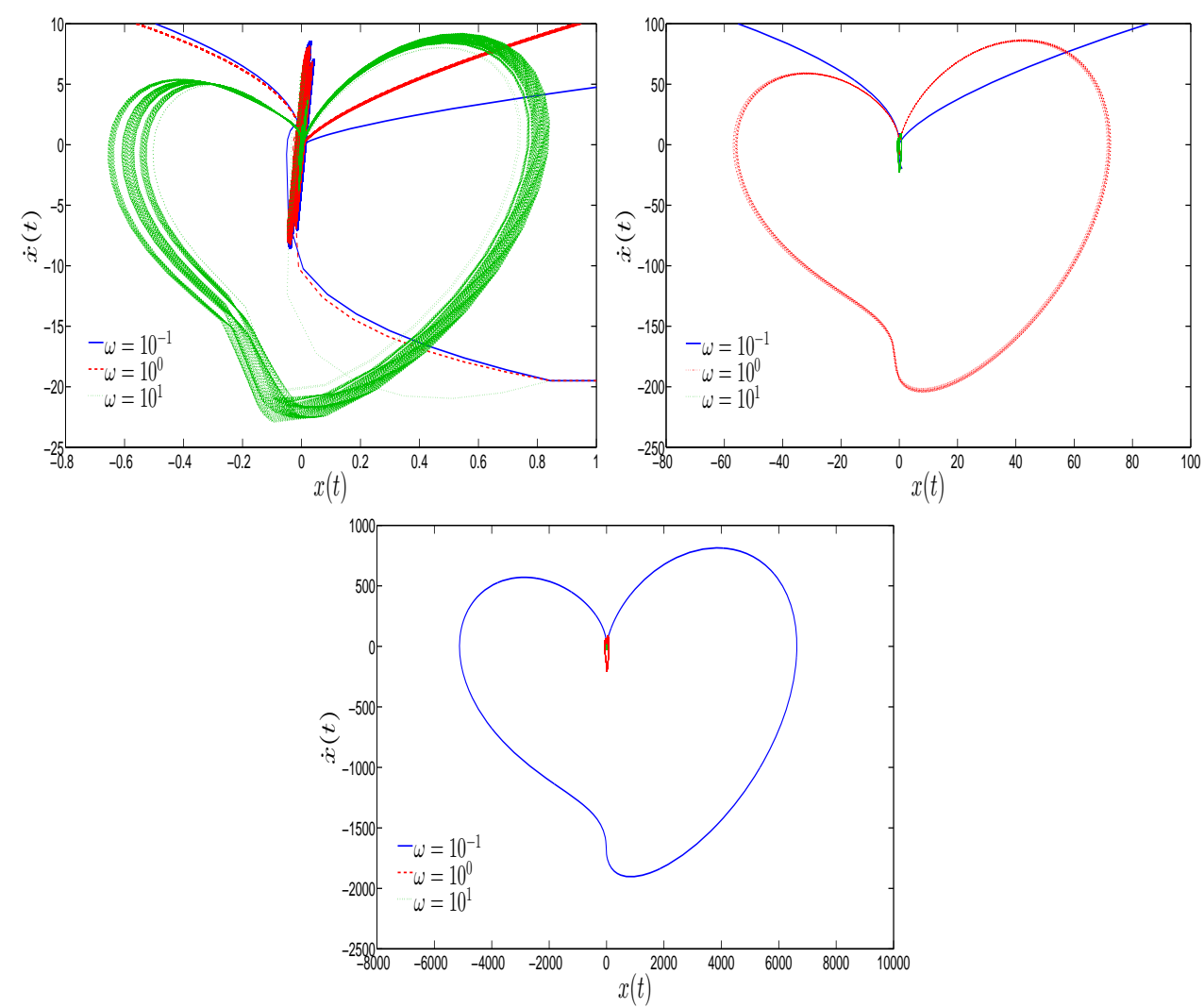

Figure 2. Trajectories $\dot{x}(t)$ vs $x(t)$ for fixed $\delta=2.5, k_{1}=20$ and $k_{2}=100$. The bottom graph depicts the trajectories of the system for three different frequencies, i.e. $\omega=0.1[\mathrm{rad} / \mathrm{s}]$ (solid blue line), $\omega=1[\mathrm{rad} / \mathrm{s}]$ (dashed red line) and $\omega=10[\mathrm{rad} / \mathrm{s}]$ (dotted green line). The right-top graph shows a zoom in the bottom graph while the left-top graph depicts another zoom.

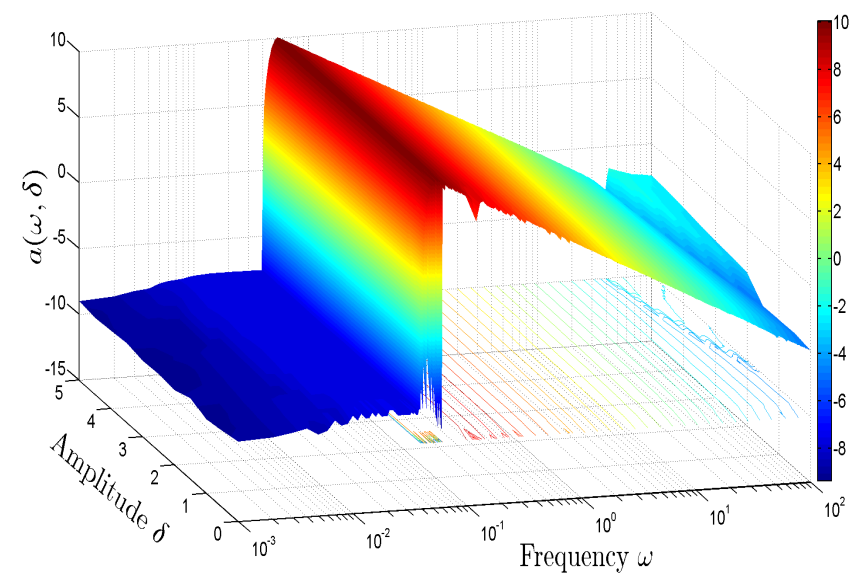

Figure 3. Map $a(\omega, \delta)=\log \left[\max _{50 \leq t \leq 100}|x(t)|\right]$ applied to the closed-loop system (6)-(7) with $d(t)=1.5 \omega \cos (\omega t) u_{1}(t)+\delta(2 \omega \cos (2 \omega t)-\omega \sin (\omega t))$, $k_{1}=20$ and $k_{2}=100$.

From Fig. 3, one concludes again that the system is converging to the origin for small values of the frequency $\omega$; and the "resonance" may happen for some values of $\omega$.

The short-finite-time stability described in Lemma 3 is depicted by Fig. 4, for some values of frequency. The results given in this figure shows that there exists $\omega_{0}>0$ such that for $\omega \in\left[-\omega_{0}, \omega_{0}\right]$ system (6)-(7) is Short-FTS with respect to certain $\left(B_{\rho}, B_{\vartheta}, T^{0}, T_{f}\right)$. Note also the existence of some trajectories which may leave the set $B_{\rho}$ due to non-uniqueness solutions of the system (6)-(7). However, after a while, the steady state $x=0$ becomes again Short-FTS with respect to another interval of time. 


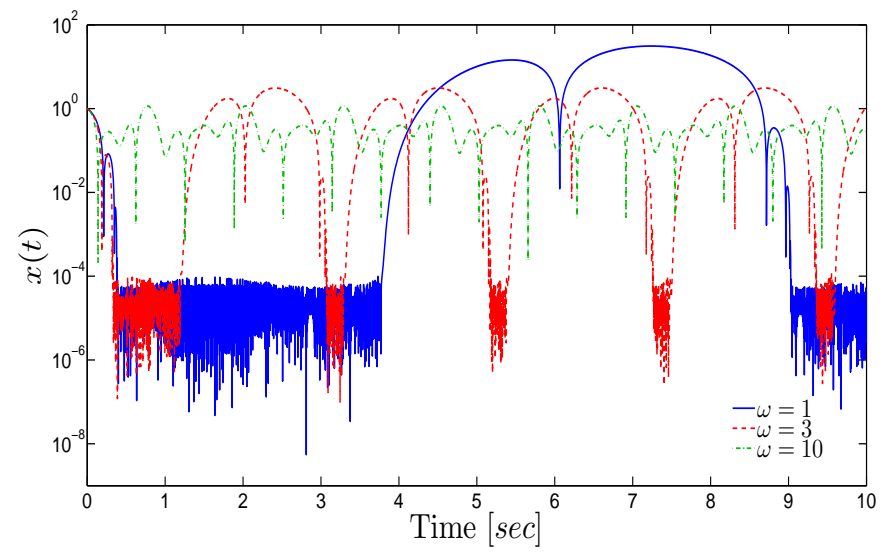

Figure 4. Illustration for Lemma 3 applied to the closed-loop system (6)-(7) with $d(t)=1.5 \omega \cos (\omega t) u_{1}(t)+\delta(2 \omega \cos (2 \omega t)-\omega \sin (\omega t))$, $\delta=2.5$, $k_{1}=20$ and $k_{2}=100$. Note that for $\omega=10[\mathrm{rad} / \mathrm{s}]$ the closed-loop system is not Short-FTS.

\section{B. Three-Tank System}

Let us consider now the three-tank system depicted by Fig. 5. The dynamical model is described as follows (see, e.g. [38], Chapter 10; and [39]):

$$
\begin{aligned}
& \dot{h}_{1}(t)=\frac{1}{A}\left(q_{P_{1}}-c_{12}\left\lceil h_{12}(t)\right\rfloor^{\frac{1}{2}}\right), \\
& \dot{h}_{2}(t)=\frac{1}{A}\left(c_{12}\left\lceil h_{12}(t)\right\rfloor^{\frac{1}{2}}-c_{23}\left\lceil h_{23}(t)\right\rfloor^{\frac{1}{2}}-q_{2}(t)\right), \\
& \dot{h}_{3}(t)=\frac{1}{A}\left(q_{P_{2}}+c_{23}\left\lceil h_{23}(t)\right\rfloor^{\frac{1}{2}}\right),
\end{aligned}
$$

where $c_{i j}$ is a constant depending on the geometry of the connecting pipe and the corresponding valve, $h_{i j}=h_{i}-h_{j}$ is the difference between the water levels $h_{i}$ and $h_{j}$, respectively. The flows $q_{P_{1}}, q_{P_{2}}$ depend on the position of the valves and it is assumed that they are constants; whilst the flow $q_{2}=c_{2} \sqrt{h_{2}}$ is the outflow to consumer. All the corresponding parameters are given in Table I.

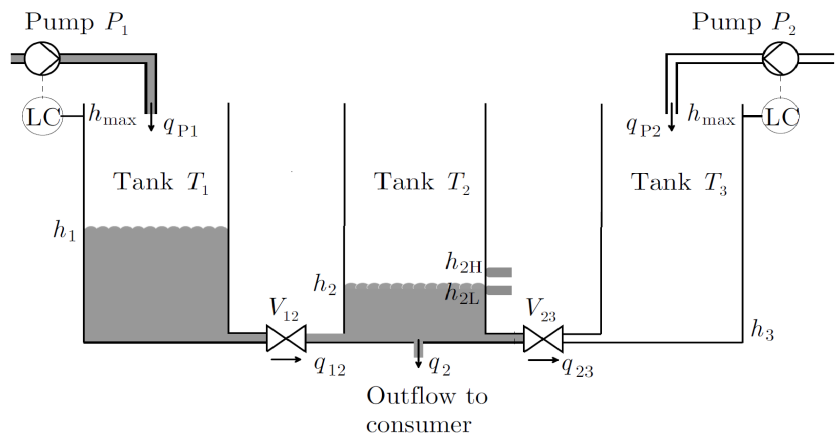

Figure 5. Three-Tank System

Consider the nominal system without inputs, i.e. $q_{P_{i}}=0, i=1,2$; then the three-tank system is $r$-homogeneous with degree $\nu=-0.5$ for $r=\left[r_{1}, r_{2}, r_{3}\right]=[1,1,1]$. Then, let us show that the nominal system without inputs is GAS. Consider the following function

$$
V\left(h_{1}, h_{2}, h_{3}\right)=h_{1}+h_{2}+h_{3} .
$$

Such a function $V$ is positive definite since $h_{i} \geq 0, i=\overline{1,3}$, and continuously differentiable with $\dot{V}\left(h_{1}, h_{2}, h_{3}\right)=-c_{2} \sqrt{h_{2}} \leq$ $-W\left(h_{1}, h_{2}, h_{3}\right)$, negative semi-definite. Note that the limit $W\left(h_{1}, h_{2}, h_{3}\right) \rightarrow 0$ implies that $h_{2}(t)$ approaches to the set $E=\left\{\left(h_{1}, h_{2}, h_{3}\right) \in \mathbb{R}^{3} \mid W\left(h_{1}, h_{2}, h_{3}\right)=0\right\}$ as $t \rightarrow \infty$. Note that $W\left(h_{1}, h_{2}, h_{3}\right)=0$ implies $h_{2}(t)=0$, and from the system dynamics, it follows that also $h_{1}(t)=0$ and $h_{3}(t)=0$. Thus, the set $E$ contains no solutions other than the trivial solution, and by LaSalle's theorem (see, e.g. [33]), global asymptotic stability is concluded. Hence, by homogeneity the three-tank system is ISS with respect to the inputs $q_{P_{i}}, i=1,2$ (see, e.g. [35]).

Define $\bar{h}_{i}(t)=h_{i}(t)-h_{\mathrm{eq}_{i}}, i=\overline{1,3}$, where $h_{\mathrm{eq}_{i}}$ is an equilibrium-point for the tank $i \in \overline{1,3}$. Then, it is easy to prove that 
Table I

VARIABLES AND PARAMETERS OF THREE-TANK SYSTEM.

\begin{tabular}{|ccc|}
\hline Variables and Parameters & Description & Unit \\
\hline \hline$h_{1}, h_{2}, h_{3}$ & Tank levels in meters & {$[\mathrm{m}]$} \\
$q_{P_{1}}, q_{P_{2}}, q_{L}, q_{2}$ & Volume flows in cubic meters per second & {$\left[\mathrm{m}^{3} / \mathrm{s}\right]$} \\
\hline$A$ & Cross-section area & $1.54 \times 10^{-2}\left[\mathrm{~m}^{2}\right]$ \\
$h_{\max }$ & Height of tanks & $0.60[\mathrm{~m}]$ \\
$c_{12}$ & Flow constant of valve $V_{12}$ & $1.6 \times 10^{-4}\left[\mathrm{~m}^{\frac{5}{2}} / \mathrm{s}\right]$ \\
$c_{23}$ & Flow constant of valve $V_{23}$ & $1.6 \times 10^{-4}\left[\mathrm{~m}^{\frac{5}{2}} / \mathrm{s}\right]$ \\
$c_{2}$ & Flow constant of the outlet of tank 2 & $1.6 \times 10^{-4}\left[\mathrm{~m}^{\frac{5}{2}} / \mathrm{s}\right]$ \\
${ }{ }_{P_{1}}$ & Flow constant of pump 1 & $1 \times 10^{-4}\left[\mathrm{~m}^{3} / \mathrm{s}\right]$ \\
${ }^{c} P_{2}$ & Flow constant of pump 2 & $1 \times 10^{-4}\left[\mathrm{~m}^{3} / \mathrm{s}\right]$ \\
\hline
\end{tabular}

the three-tank system is GAS with respect to any equilibrium-point; and consequently, it is also ISS with respect to the inputs $q_{P_{i}}, i=1,2$, for its particular equilibrium-point.

Now, let us apply the results established in Theorem 2 in order to prove the short-finite-time stability of the three-tank system with respect to some time-varying perturbations.

Consider that all the flow constants of the corresponding valves are affected by a time-varying perturbation $d(t)$, i.e. $c_{i j}(t)=(d(t)+1) c_{i j}$ (for brevity, the same perturbation is considered for all the coefficients). Thus, for the system without inputs, the conditions of Theorem 1 are satisfied with $r=[1,1,1], \tilde{r}=0$ (the weight corresponding to $d$ ), and $\nu=-0.5$. Then, for all the time-varying perturbations satisfying $d \in \mathcal{L}_{\varrho, \delta}^{1}$, and $d(t)=d(t+\mathcal{T})$, for all $t \in \mathbb{R}$, with $d(t) \neq 0$ and some $\mathcal{T}>0$, one can conclude by Theorem 2 that the three-tank system is Short-FTS with respect to any equilibrium-point. For the case in which the flows $q_{P_{i}}, i=1,2$ are viewed as perturbations, one gets that $r=[1,1,1], \tilde{r}=[0.5,0.5,0]$ (the weights corresponding to $q_{P_{1}}, q_{P_{2}}$, and $d$ ), and then based on Theorem 1, only iISS with respect to its particular equilibrium-point is concluded.

Let us consider $q_{P_{i}}=1 \times 10^{-4}, i=1,2, d(t)=\delta(1-\cos (\omega t)) \in \mathcal{L}_{\varrho, 2 \delta}^{1}$, with $\varrho(s)=s$; initial state $h(0)=[0.3,0.2,0.1]^{T}$, equilibrium-point $h_{\mathrm{eq}}=[0.3906,0,0.3906]^{T}$, and the parameters given in Table I. The three-tank system was simulated on the time interval $[0,500]$ for different values of $(\omega, \delta)$. The results given by Fig. 6 depict the behavior of $a(\omega, \delta)$ from which one concludes that the "resonance" may happen for particular values of $\omega$ and $\delta$.

Finally, the results depicted in Fig. 7 show the integral input-to-state stability described in Theorem 1, for different values of frequency. The results show that there exists $\omega_{0}>0$ such that for $\omega \in\left[-\omega_{0}, \omega_{0}\right]$ the three-tank system is Short-TS with respect to certain $\left(B_{\rho}, B_{\vartheta \rho}, T^{0}, T_{f}\right)$.

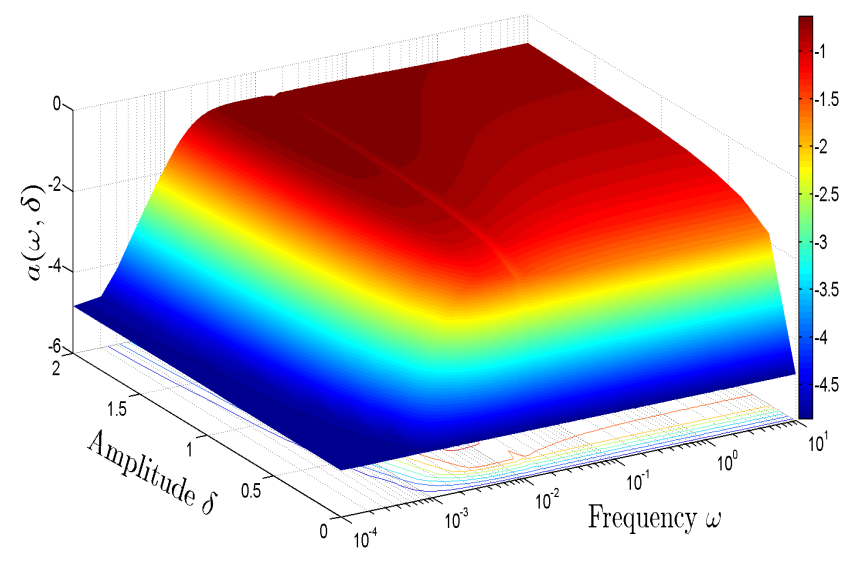

Figure 6. Map $a(\omega, \delta)=\log \left[\max _{300 \leq t \leq 500}|\bar{h}(t)|\right]$ applied to three-tank system with $d(t)=\delta(1-\cos (\omega t))$.

\section{CONCLusions}

In this paper the problem of stability robustness with respect to time-varying perturbations of a given frequency spectrum is studied applying homogeneity framework. The notion of short-finite-time stability is introduced and used for that purpose. The results are applied to analyze the Super-Twisting algorithm behavior under some time-varying perturbations, and to demonstrate some robustness properties of the three-tank system. Some simulation examples illustrated these robustness properties. Application of the developed results on robustness for designing of control, estimation or identification algorithms in time-varying systems is a direction of future research. 


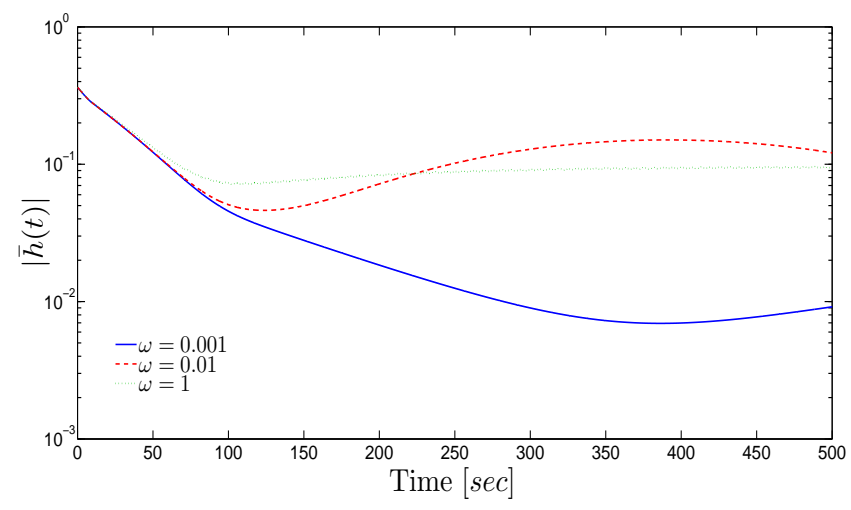

Figure 7. Illustration for iISS properties applied to the three-tank system with $d(t)=\delta(1-\cos (\omega t)), \delta=0.1$.

\section{ACKNOWLEDGMENT}

This work was supported in part by the Government of Russian Federation (Grant 074-U01) and the Ministry of Education and Science of Russian Federation (Project 14.Z50.31.0031).

\section{REFERENCES}

[1] A. Bacciotti and L. Rosier, Liapunov Functions and Stability in Control Theory, vol. 267 of Lecture Notes in Control and Inform. Sci. Berlin: Springer, 2001.

[2] V. Zubov, "On systems of ordinary differential equations with generalized homogenous right-hand sides," Izvestia vuzov. Mathematica., vol. 1, pp. 80-88, 1958. in Russian.

[3] L. Rosier, "Homogeneous Lyapunov function for homogeneous continuous vector field," Systems\&Control Lett., vol. 19, pp. 467-473, 1992.

[4] D. Efimov and W. Perruquetti, "Oscillations conditions in homogenous systems," in Proc. NOLCOS'10, (Bologna), pp. 1379-1384, 2010.

[5] V. Andrieu, L. Praly, and A. Astolfi, "Homogeneous approximation, recursive observer design, and output feedback," SIAM J. Control Optimization, vol. 47, no. 4, pp. 1814-1850, 2008.

[6] H. Hermes, Differential Equations: Stability and Control, vol. 109 of Lecture Notes in Pure Appl. Math., ch. Homogeneous coordinates and continuous asymptotically stabilizing feedback controls, pp. 249-260. Marcel Dekker, 1991.

[7] Y. Hong, "Finite-time stabilization and stabilizability of a class of controllable systems," Systems\&Control Lett., vol. 46, pp. 231-236, 2002.

[8] H. Hermes, "Nilpotent and high-order approximations of vector field systems," SIAM Review, vol. 33, no. 2, pp. 238-264, 1991.

[9] T. Ménard, E. Moulay, and W. Perruquetti, "Homogeneous approximations and local observer design," ESAIM: Control, Optimization and Calculus of Variations, vol. 19, no. 3, pp. 906-929, 2013.

[10] S. Bhat and D. Bernstein, "Geometric homogeneity with applications to finite-time stability," Mathematics of Control, Signals and Systems, vol. 17, pp. 101-127, 2005.

[11] L. Grüne, "Homogeneous state feedback stabilization of homogeneous systems," SIAM J. Control Optimization, vol. 38, no. 4, pp. 1288-1314, 2000.

[12] M. Kawski, Homogeneous feedback stabilization, vol. 7 of Progress in systems and control theory: New trends in systems theory. Birkhäuser, 1991.

[13] E. Moulay and W. Perruquetti, "Finite time stability and stabilization of a class of continuous systems," J. Mathematical Analysis Applications, vol. 323, no. 2, pp. 1430-1443, 2006.

[14] R. Sepulchre and D. Aeyels, "Stabilizability does not imply homogeneous stabilizability for controllable systems," SIAM J. Control Optimization, vol. 34, no. 5, pp. 1798-1813, 1996.

[15] A. Polyakov, D. Efimov, and W. Perruquetti, "Homogeneous differentiator design using implicit Lyapunov function method," in Proc. European Control Conference (ECC), (Strasbourg), 2014.

[16] E. Ryan, "Universal stabilization of a class of nonlinear systems with homogeneous vector fields," Systems \& Control Letters, vol. 26, pp. 177-184, 1995.

[17] Y. Hong, "H $\mathrm{H}_{\infty}$ control, stabilization, and input-output stability of nonlinear systems with homogeneous properties," Automatica, vol. 37, no. 7, pp. 819$829,2001$.

[18] E. Bernuau, A. Polyakov, D. Efimov, and W. Perruquetti, "On ISS and iISS properties of homogeneous systems," in Proc. European Control Conference (ECC) 2013, (Zürich), 2013.

[19] E. Bernuau, A. Polyakov, D. Efimov, and W. Perruquetti, "Verification of ISS, iISS and IOSS properties applying weighted homogeneity," Systems \& Control Letters, vol. 62, no. 12, pp. 1159-1167, 2013

[20] J. Peuteman and D. Aeyels, "Averafing results and the study of uniform asymptotic stability of homogeneous differential equations that are not fast time-varying," SIAM J. Control Optim., vol. 37, no. 4, pp. 997-1010, 1999.

[21] Y. Orlov, "Finite time stability and robust control synthesis of uncertain switched systems," SIAM J. Control Optim., vol. 43, no. 4, pp. 1253-1271, 2005.

[22] H. Ríos, D. Efimov, L. Fridman, J. Moreno, and W. Perruquetti, "Homogeneity based uniform stability analysis for time-varying systems," IEEE Transactions on Automatic Control, 2015. DOI: 10.1109/TAC.2015.2446371.

[23] E. Roxin, "On finite stability in control systems," Rendiconti del Circolo Matematico di Palermo, vol. 15, pp. 273-283, 1966.

[24] P. Dorato, "An overview of finite-time stability," in Current Trends in Nonlinear Systems and Control (L. Menini, L. Zaccarian, and C. T. Abdallah, eds.), Systems \& Control: Foundations \& Applications, pp. 185-194, Boston: Birkhäuser, 2006.

[25] S. Nersesov, W. Haddad, and Q. Hui, "Finite-time stabilization of nonlinear dynamical systems via control vector Lyapunov functions," J. Franklin Inst., vol. 345, pp. 819-837, 2008.

[26] W. M. Haddad, S. G. Nersesov, and L. Du, "Finite-time stability for time-varying nonlinear dynamical systems," in Proc. ACC, (Seattle), pp. 4135-4139, 2008.

[27] A. Polyakov, "Nonlinear feedback design for fixed-time stabilization of linear control systems," IEEE Transactions on Automatic Control, vol. 57, no. 8, pp. 2106-2110, 2012.

[28] E. Cruz-Zavala, J. Moreno, and L. Fridman, "Uniform robust exact differentiator," IEEE Transactions on Automatic Control, vol. 56, no. 11, pp. 2727$2733,2011$. 
[29] G. Kamenkov, "On stability of motion over a finite interval of time," Journal of Applied Math. and Mechanics (PMM), vol. 17, pp. 529-540, 1953.

[30] A. Lebedev, "The problem of stability in a finite interval of time," Journal of Applied Math. and Mechanics (PMM), vol. 18, pp. 75-94, 1954.

[31] P. Dorato, Short-time stability in linear time-varying systems. PhD thesis, Polytechnic Institute of Brooklyn, New York, 1961.

[32] L. Weiss and E. Infante, "On the stability of systems defined over a finite time interval," Proc. of the National Academy of Sciences, vol. 54, pp. 440-448, 1965.

[33] H. Khalil, Nonlinear Systems. Upper Saddle River, New Jersey: Prentice Hall, 3rd ed., 2002.

[34] W. Perruquetti, J. Richard, L. Grujić, and P. Borne, "On practical stability with the settling time via vector norms," International Journal of Control, vol. 62, no. 1, pp. 173-189, 1995.

[35] E. Bernuau, D. Efimov, W. Perruquetti, and A. Polyakov, "On an extension of homogeneity notion for differential inclusions," in 2013 European Control Conference, (Zürich, Switzerland), pp. 2204-2209, 2013.

[36] A. Levant, "Robust exact differentiation via sliding mode technique," Automatica, vol. 34, no. 3, pp. 379-384, 1998.

[37] J. Moreno and M. Osorio, "Strict Lyapunov functions for Super-Twisting algorithm," IEEE Transactions on Automatic Control, vol. 57, no. 4, pp. 1035$1040,2006$.

[38] M. Blanke, M. Kinnaert, J. Lunze, and M. Staroswiecki, Diagnosis and fault tolerant control. New York: Springer, 2003.

[39] Documentation of the three-tank system. 65, D-47057 Duisburg, Germany: Bismarckstr Amira GmbH, 1994.

\section{APPENDIX}

The following auxiliary result is required.

Claim 1. Let the system (5) be $r$-homogeneous with degree $\nu \neq 0$, asymptotically stable for $\omega=0$, and Assumption 1 be satisfied. Then for any $\rho>\varepsilon>0$ and any $T^{0}>0$, there exists $\omega^{T^{0}, \rho, \varepsilon}>0$ such that $\left|x\left(T^{T^{0}, \rho, \varepsilon}\right)\right|_{r} \leq \varepsilon$ for some $T^{T^{0}, \rho, \varepsilon} \in\left(t_{0},+\infty\right)$ for all solutions of (5) with $|\omega| \leq \omega^{T^{0}, \rho, \varepsilon}$ provided that initial states, at $t_{0} \in\left[-T^{0}, T^{0}\right]$, satisfy $x\left(t_{0}\right) \in \rho \mathbb{S}_{r}$.

Proof. By conditions of the claim, the time-invariant modification of (5),

$$
\frac{d x(t)}{d t}=f(0, x(t))
$$

is globally asymptotically stable and $r$-homogeneous. Then there is a homogeneous Lyapunov function $V: \mathbb{R}^{n} \rightarrow \mathbb{R}_{+}$of degree $\mu>0$ such that:

$$
\begin{gathered}
a=-\sup _{\xi \in \mathbb{S}_{r}} \frac{d V(\xi)}{d \xi} f(0, \xi)>0, \\
0<b=\sup _{\xi \in \mathbb{S}_{r}}\left|\frac{\partial V(\xi)}{\partial \xi}\right|<+\infty, \\
c_{1}=\inf _{\xi \in \mathbb{S}_{r}} V(\xi), c_{2}=\sup _{\xi \in \mathbb{S}_{r}} V(\xi), \\
c_{1}|x|_{r}^{\mu} \leq V(x) \leq c_{2}|x|_{r}^{\mu} \quad \forall x \in \mathbb{R}^{n} .
\end{gathered}
$$

Take $x \in \mathbb{R}^{n}$, then there is $\xi \in \mathbb{S}_{r}$ such that $x=\Lambda_{r}(\lambda) \xi$ for $\lambda=|x|_{r}$. Let us derive the derivative of $V$ for the system in (5):

$$
\begin{aligned}
\dot{V}= & \frac{d V(x)}{d x} f(\omega t, x)=\frac{d V(x)}{d x} f(0, x) \\
& +\frac{d V(x)}{d x}[f(\omega t, x)-f(0, x)] \\
= & \lambda^{\nu+\mu}\left\{\frac{d V(\xi)}{d \xi} f(0, \xi)+\frac{d V(\xi)}{d \xi}[f(\omega t, \xi)-f(0, \xi)]\right\} \\
\leq & \lambda^{\nu+\mu}\{-a+b|f(\omega t, \xi)-f(0, \xi)|\} \\
\leq & |x|_{r}^{\nu+\mu}\{-a+b \sigma(|\omega t|)\} .
\end{aligned}
$$

For $|t| \leq T_{\omega}=|\omega|^{-1} \sigma^{-1}\left(0.5 a b^{-1}\right)$, it is obtained:

$$
\dot{V} \leq-0.5 a|x|_{r}^{\nu+\mu} \leq-0.5 a c_{2}^{-1-\nu / \mu} V^{1+\nu / \mu} .
$$

If $t_{0} \in\left(-T_{\omega}, T_{\omega}\right)$, then for $t \in\left[t_{0}, T_{\omega}\right]$, it follows that

$$
V(t) \leq\left[V\left(t_{0}\right)^{-\nu / \mu}+0.5 a c_{2}^{-1-\nu / \mu} \nu \mu^{-1}\left(t-t_{0}\right)\right]^{-\mu / \nu},
$$

and

$$
|x(t)|_{r} \leq c_{1}^{-1} c_{2}^{1 / \mu}\left[\left|x\left(t_{0}\right)\right|_{r}^{-\nu}+0.5 a c_{2}^{-1} \nu \mu^{-1}\left(t-t_{0}\right)\right]^{-1 / \nu} .
$$

From this expression, if $t \geq T^{t_{0}, \rho, \varepsilon}=t_{0}+\frac{c_{1}^{-\nu} c_{2}^{\nu / \mu} \varepsilon^{-\nu}-\rho^{-\nu}}{0.5 a c_{2}^{-1} \nu \mu^{-1}}$, then $|x(t)|_{r} \leq \varepsilon$ provided that $\left|x\left(t_{0}\right)\right|_{r}=\rho$. Therefore, for any $t_{0} \in\left[-T^{0}, T^{0}\right]$ (if the conclusion above is satisfied for the initial time $T^{0}$, then the same is true for all $\left|t_{0}\right| \leq T^{0}$ ), all solutions of (5) with $|\omega| \leq \omega^{T^{0}, \rho, \varepsilon}=\frac{\sigma^{-1}\left(0.5 a b^{-1}\right)}{T^{T^{0}, \rho, \varepsilon}}$ and initial states from the sphere $\rho \mathbb{S}_{r}$, for any $\rho>0$, reach the ball $B_{\epsilon}$, in a finite time less than $T^{T^{0}, \rho, \varepsilon}$. 
In the claim proof the following expressions for $T^{T^{0}, \rho, \varepsilon}$ and $\omega^{T^{0}, \rho, \varepsilon}$ have been established:

$$
\begin{aligned}
T^{T^{0}, \rho, \varepsilon} & =T^{0}+\frac{c_{1}^{-\nu} c_{2}^{\nu / \mu} \varepsilon^{-\nu}-\rho^{-\nu}}{0.5 a c_{2}^{-1} \nu \mu^{-1}}, \\
\omega^{T^{0}, \rho, \varepsilon} & =\frac{\sigma^{-1}\left(0.5 a b^{-1}\right)}{T^{T^{0}, \rho, \varepsilon}},
\end{aligned}
$$

where the meaning of parameters $a, b, \mu, c_{1}$ and $c_{2}$ is explained above (they all are related with the properties of the timeinvariant system (5) with $t=0$ ).

\section{Proof of Lemma 2}

The conditions of Claim 1 are satisfied. Note that for $\nu>0$

$$
\begin{aligned}
T^{T^{0},+\infty, \rho} & =T^{0}+2 \frac{\mu c_{2}^{1+\nu / \mu}}{a \nu c_{1}^{\nu} \rho^{\nu}}, \\
\omega^{T^{0},+\infty, \rho} & =\frac{\sigma^{-1}\left(0.5 a b^{-1}\right)}{T^{T^{0},+\infty, \rho}}
\end{aligned}
$$

are finite constants for any $\rho>0$ and $T^{0}>0$. Let us fix $\rho>0$ and $T^{0}>0$ and consider a solution of (5) for $|\omega| \leq \omega_{0}=$ $\omega^{T^{0}},+\infty, \rho$ with initial state $x_{0} \notin B_{\rho}$ at the instant $t_{0} \in\left[-T^{0}, T^{0}\right]$. From Claim $1,\left|x\left(T^{T^{0},+\infty, \rho}, t_{0}, x_{0}\right)\right|_{r} \leq \rho$, that is necessary to prove.

\section{Proof of Lemma 3}

The conditions of Claim 1 are satisfied. For $\nu<0$, it is given that

$$
\begin{aligned}
& T^{T^{0}, \rho, 0}=T^{0}-\frac{c_{2} \mu \rho^{-\nu}}{0.5 a \nu}, \\
& \omega^{T^{0}, \rho, 0}=\frac{\sigma^{-1}\left(0.5 a b^{-1}\right)}{T^{T^{0}, \rho, 0}}
\end{aligned}
$$

are finite constants for any $\rho>0$ and $T^{0}>0$. Let us fix $\rho>0$ and $T^{0}>0$ and consider a solution of (5) for $|\omega| \leq \omega_{0}=$ $\frac{\sigma^{-1}\left(0.5 a b^{-1}\right)}{T^{T^{0}, \rho, 0}}$ with initial state $x_{0} \in B_{\rho}$ at the instant $t_{0} \in\left[-T^{0}, T^{0}\right]$, then $\left|x\left(T^{T^{0}, \rho, 0}, t_{0}, x_{0}\right)\right|_{r}=0$ from Claim 1 . According

to proof of Claim $1, \dot{V}(t) \leq 0$ for all $T^{T^{0}, \rho, 0} \geq t \geq t_{0} \in\left[-T^{0}, T^{0}\right]$, that implies short-finite-time stability of (5) for $|\omega| \leq \omega_{0}$ with respect to $\left(B_{\rho}, B_{\vartheta \rho}, T^{0}, T^{T^{0}, \rho, 0}\right)$, where $\vartheta=c_{1}^{-1 / \mu} c_{2}^{1 / \mu}$.

\section{Proof of Theorem 2}

Since conditions of Theorem 1 are satisfied for (4) with $\nu<0$ and $\tilde{r}_{\min }=0$, then (4) is iISS and there exist a function $\gamma \in \mathcal{K}_{\infty}$ (related with asymptotic gain of (4)) such that for all $x_{0} \in \mathbb{R}^{n}$ and all $d \in \mathcal{L}_{\varrho, \delta}^{m}$ for any $\varrho \in \mathcal{K}$ and any $t_{0} \in \mathbb{R}$ :

$$
\left|x\left(t, t_{0}, x_{0}\right)\right|_{r} \leq \gamma(\delta) \quad \forall t \geq t_{0}+T^{\delta, x_{0}}, T^{\delta, x_{0}} \geq 0,
$$

where the homogeneous norm is used in the right-hand side due to the norm equivalence (2).

Under theorem conditions the system (4) is $r$-homogeneous in the sense of Definition 4 for

$$
f(t, x)=F(d(t), x),
$$

and for such an $f$ the system (5) is asymptotically stable for $t=0$ due to $d(0)=0$ (it is assumed that $d \in \mathcal{L}_{\varrho, \delta}^{m}$ ). To verify Assumption 1 consider:

$$
\begin{gathered}
\sup _{\xi \in \mathbb{S}_{r}}|f(\tau, \xi)-f(0, \xi)|=\sup _{\xi \in \mathbb{S}_{r}}|F(d(\tau), \xi)-F(d(0), \xi)| \\
\leq \varsigma \circ \varrho(|\tau|)=\sigma(|\tau|),
\end{gathered}
$$

where existence of such a function $\varsigma \in \mathcal{K}_{\infty}$ follows from continuity of $F$ and existence of $\varrho$ is fixed for $d \in \mathcal{L}_{\varrho, \delta}^{m}$. Therefore, all conditions of Lemma 3 are satisfied and the input $d(t)$ is periodic with the period $\mathcal{T}$, then for any $t_{0} \in[-0.5 \mathcal{T}, 0.5 \mathcal{T}]$ and any $\rho=\gamma(\delta)$ there is $\varrho \in \mathcal{K}$ sufficiently small (this gain function plays the role of the parameter $\omega$ in Lemma 3) such that (4) with $d \in \mathcal{L}_{\varrho, \delta}^{m}$ is short-finite-time stable with respect to $\left(B_{\rho}, B_{\vartheta \rho}, 0.5 \mathcal{T}, T_{f}\right)$ for some $\vartheta>1$ and $T_{f}>0.5 \mathcal{T}$ (the time $T_{f}$ depends on $\rho=\gamma(\delta)$ and $\mathcal{T}$ ). Combining this result with iISS property established previously, since $t_{0}+T^{\delta, x_{0}}$ can be projected into $[-0.5 \mathcal{T}, 0.5 \mathcal{T}]$, due to its periodicity; the required conclusion is obtained. 


\section{Proof of Corollary 5}

It is straightforward to verify, that the system

$$
\dot{x}(t) \leq-\alpha x^{\eta}(t)+k(\omega t) x^{\eta}(t),
$$

which is a comparison system for the Lyapunov function $V$ with $x\left(t_{0}\right)=V\left(t_{0}, x\left(t_{0}\right)\right)$, satisfies all conditions Theorem 2 and Lemma 3 for $\nu=\eta-1<0$. The result of this corollary follows Lemma 3 for the case $\Omega \subset \mathbb{R}^{n}$ and Theorem 2 for the case $\Omega=\mathbb{R}^{n}$. 